\title{
Do encantado da floresta ao fantástico da escola: cosmologia Ticuna e as árvores das crianças ${ }^{1}$
}

\author{
Del encanto del bosque al fantástico de la escuela: cosmología Ticuna y \\ los árboles de los niños
}

\author{
From the enchanted forest to the fantastic of the school: Ticuna cosmology \\ and the children's trees
}

\author{
Clarissa Lopes Suzuki² \\ Eliene de Oliveira Aleixo ${ }^{3}$
}

\begin{abstract}
Resumo
Este texto é um relato de experiências vivenciadas em um trabalho realizado no ano de 2014, na EMEF Desembargador Amorim Lima, escola da rede municipal da cidade de São Paulo. O projeto "Árvores e outras plantas do Amorim" foi desenvolvido com crianças de 6 e 7 anos, como parte dos estudos da Festa da Cultura, que tinha como tema "Os Povos Originários do Brasil". Em consonância com a Lei 11.645/08, que altera a LDB 9394/96, tendo em vista uma aprendizagem significativa, o cuidado fundamental na realização do trabalho era não reproduzir estereótipos conservadores, que generalizam saberes e fazeres de culturas diversas e historicamente homogeneizadas, como aqueles encontrados nos livros didáticos estruturados pela lógica ocidental moderna. Nesse sentido, os contos Ticuna de O Livro das Árvores, organizado pelos Professores Ticuna Bilingues do Amazonas, que apresenta a cosmologia da etnia, inspirou o projeto, cujo objeto era reconhecer as árvores da escola, componentes essenciais nas brincadeiras diárias das crianças, disparando, dessa forma, a construção de um percurso de aprendizagem que não separa a razão da emoção. Para isso, em diálogo com as relações afetivas estabelecidas entre as crianças e as árvores da escola, foram experienciadas práticas de observação, nomeação e criação do povo Ticuna, o que desencadeou estudos de etnobotânica em tupi, cartografias das árvores, desenhos de observação, produções oral, visual e escrita e, por fim, a composição de um livro registrando todo o processo de aprendizagem. A experiência permitiu conhecer a complexidade da cosmologia Ticuna, desconstruindo a ideia do colonizador, quando afirma que "índio é tudo igual", ao mesmo tempo que promove a valorização do conhecimento que se estrutura a partir das vivências cotidianas das crianças.
\end{abstract}

Palavras-chave: Decolonização do Currículo; Diversidade Cultural; Escola Pública; Lei 11.645/08; Povos Originários.

\section{Resumen}

Este texto es un relato de experiencias vividas en un trabajo realizado en el año 2014, en la EMEF Desembargador Amorim Lima, escuela de la red municipal de la ciudad de São Paulo. El proyecto "Árboles y otras plantas de Amorim" fue desarrollado con niños de 6 y 7 años, como parte de los estudios de la Fiesta de la Cultura, que tenía como tema "Los Pueblos Originarios de Brasil".

En consonancia con la Ley 11.645/08, que altera la LDB 9394/96, con vistas a un aprendizaje significativo, el cuidado fundamental en la realización del trabajo era no reproducir estereotipos conservadores, que generalizan

\footnotetext{
${ }^{1}$ Artigo apresentado no Simpósio Temático Políticas públicas, diversidade cultural e descolonização durante o II Seminário Latino-Americano de Estudos em Cultura - SEMLACult em Foz do Iguaçu/PR, Brasil, 2018.

${ }^{2}$ Doutoranda em Artes Visuais na USP; Escola de Comunicações e Artes da USP (ECA/USP); São Paulo, São Paulo, Brasil; clarissasuzuki@usp.br.

${ }^{3}$ Especialista em Ética, Valores e Cidadania pela USP; EMEF Desembargador Amorim Lima; São Paulo, São Paulo, Brasil; elieneoliveiraaleixo@gmail.com.
} 
el saber y hacer de las culturas diversas e históricamente homogeneizadas, como los que se encuentran en los libros didácticos estructurados por la lógica occidental moderna.

En este sentido, los cuentos Ticuna del El libro de los árboles organizado por los Profesores Ticuna Bilingües del Amazonas, que presenta la cosmología de la etnia, inspiró el proyecto con los árboles de la escuela, que forman parte de los juegos de los niños, comenzando la construcción de un recorrido de aprendizaje que no separa la razón de la emoción. Para eso, el diálogo con las relaciones afectivas establecidas entre los niños y los árboles de la escuela, fueron vivenciadas prácticas de observación, nombramiento y creación del pueblo Ticuna, lo que resultó en estudios de etnobotánica en tupí, cartografías de los árboles, dibujos de observación, producciones orales, visuales y escritas y, por fin, la presentación de un libro que muestra todo el proceso de aprendizaje. La experiencia permitió conocer la complejidad de la cosmología Ticuna, desconstruyendo la idea del colonizador de que "los indios son todo iguales" y valorar el conocimiento que se estructura desde de las vivencias cotidianas de los niños.

Palabras-clave: Decolonización del Currículo; Diversidad Cultural; Escuela Pública; Ley 11.645/08; Pueblos Originarios.

\begin{abstract}
This text is about the experiences lived in a project carried out in 2014 at the Elementary School "Desembargador Amorim Lima", which belongs to the city of São Paulo's public network. The project "Trees and other plants of Amorim" was developed with children of 6 and 7 years of age as part of the studies for the Festival of Culture, which had as its theme "Native Peoples of Brazil". In line with the Law 11.645 / 08, which amends LDB 9394/96, and in order to achieve a meaningful learning, the fundamental care in the project was to not reproduce conservative stereotypes that generalize the knowledge and practices of diverse and historically homogenized cultures, such as those found in didactic books structured by the modern Western logic. In that sense, the Ticuna tales of "The Book of Trees", organized by the Ticuna Bilingual Teachers of the Amazon that present the ethnology of ethnicity - inspired the project, whose object was to recognize the trees within the school, which are an essential component in the children's daily games, thus creating the opportunity to the construction of a learning path that does not separate reason from emotion. To this end, in dialogue with the affective relationships established between the children and the school trees, the Ticuna people's practices of observation, naming and creation were experienced, triggering studies of Etnobotany in Tupi language, tree cartography, observation drawings, a variety of productions - visual, written and spoken - and, finally, the composition of a book recording the entire learning process. The experience allowed us to learn about the complexity of the Ticuna cosmology, deconstructing the idea of the colonizer which claimed that "Indigenous peoples are all the same", while at the same time promoted the appreciation of the knowledge that is structured from the daily experiences of the children.
\end{abstract}

Keywords: Cultural diversity; Decolonization of the Curriculum; Law 11.645/08; Original Peoples; Public School.

Este texto é um relato de experiências vivenciadas em um trabalho realizado no ano de 2014 na EMEF Desembargador Amorim Lima ${ }^{4}$, escola da rede municipal da cidade de São Paulo que, desde 2004, possui um projeto pedagógico emancipador, e que, em vista disso, quebrou fisicamente e simbolicamente suas paredes, apostando na construção de um cotidiano estruturado pelo diálogo, formando uma comunidade atuante, que adota práticas inovadoras de educação ao priorizar valores como democracia, solidariedade, autonomia dos estudantes e educadores e, sobretudo, a diversidade cultural.

O trabalho foi realizado como parte dos estudos para a mostra da Festa da Cultura da escola no ano de 2014, que tinha como tema "Os Povos Originários do Brasil". Vale ressaltar

${ }^{4}$ Disponível em: 〈https://amorimlima.org.br/>. Acesso em: 16 mai.2019. 
que a escolha do tema da festa é decidido coletivamente nas instâncias de decisão da escola, como o Conselho de Escola e a Assembleia dos Estudantes. Duas turmas do primeiro ano do Ensino Fundamental I da EMEF Amorim Lima embarcaram na proposta, acompanhadas pelas professoras Eliene de Oliveira Aleixo e Maria Lucia Padilha, que trabalhavam exercitando um dos princípios pedagógicos do projeto que é a "prática compartilhada e solidária, visto que o professor não trabalha mais intramuros, solitariamente e com uma turma específica"5.

O recorte temático partiu da seguinte reflexão: O que abordar com as crianças de 6 e 7 anos sobre os saberes dos povos originários do Brasil? Grafismos, cosmologias, diversidade étnica, artesanato, brincadeiras, palavras e hábitos herdados, entre outros, foram alguns dos temas levantados pelas educadoras, a partir de suas experiências e do diálogo com os estudantes. Por meio das falas que revelaram as estruturas dos imaginários, foi possível mapear os temas que manifestavam representações homogêneas e estereotipadas as quais reproduziam visões equivocadas, como, por exemplo, a imagem de um "índio" com uma saia de penas e um cocar com uma única pena, concepções que desconsideram a diversidade étnica e reforçam a ideia de que os povos indígenas viveram no passado, portanto, invisibilizados no cotidiano. Podemos definir essas representações como formas de domesticação dos seres e dos saberes em decorrência de uma colonialidade que sobrevive através dos espaços institucionalizados, "manuais de aprendizagem, nos critérios para os trabalhos acadêmicos, na cultura, no senso comum, na autoimagem dos povos, nas aspirações dos sujeitos, e em tantos aspectos de nossa experiência moderna" (Maldonado-Torres, 2007, p.131).

A colonialidade molda um jeito de ser, fazer e se relacionar orientado por uma única forma de existir no mundo, desconsiderando as outras formas que não se assemelham à racionalidade hegemônica ocidental. Colonialidade representa, apesar do fim do colonialismo,

Um padrão de poder que emergiu como resultado do colonialismo moderno, porém, ao invés de estar limitado a uma relação formal de poder entre os povos ou nações, refere-se à forma como o trabalho, o conhecimento, a autoridade e as relações intersubjetivas se articulam entre si através do mercado capitalista mundial e da ideia de raça. (idem)

${ }^{5}$ Texto extraído do Projeto Político Pedagógico EMEF Amorim Lima. Disponível em: <https://amorimlima.org.br/institucional/projeto-politico-pedagogico/>. Acesso em: 20 jul. 2018. 
Foi nesse sentido que a valorização da cosmologia Ticuna - considerando as especificidades culturais dos diversos povos originários - foi priorizada. O Livro das Árvores Ticuna foi o disparador de ideias no desenvolvimento do projeto. A leitura do mito "A samaumeira que escurecia o mundo" teve como objetivo sensibilizar o grupo para a saída pedagógica à exposição “Amazônia Mundi”, no SESC Itaquera/SP, mas, de fato, tornou-se a referência para variadas propostas ao longo do ano, como, por exemplo, o contato com mapas cartográficos para observação e localização de regiões e povos indígenas, assim como a escrita de palavras de origem indígena que fazem parte do cotidiano (nome de animais, lugares e objetos), leituras de mitos de origem de outros povos e de outras histórias do povo Ticuna.

Abaixo segue a transcrição do mito Ticuna "A samaumeira que escurecia o mundo":

No princípio, estava tudo escuro, sempre frio e sempre noite. Uma enorme samaumeira, wotchine, fechava o mundo, e por isso não entrava claridade $\mathrm{n}$ a terra. Yo'i e Ipi ficaram preocupados. Tinham que fazer alguma coisa. Pegaram um caroço de araratucupi, tcha, e atiraram na árvore para ver se existia luz do outro lado. Através de um buraquinho, os irmãos enxergaram uma preguiça-real que prendia lá no céu os galhos da samaumeira. Jogaram muitos e muitos caroços e assim criaram as estrelas. Mas ainda não havia claridade. Yo'i e Ipi ficaram pensando e decidiram convidar todos os animais da mata para ajudarem a derrubar a árvore. Mas nenhum deles conseguiu, nem o pica-pau. Resolveram, então, oferecer a irmã Aicüna em casamento para quem jogasse formigas-de-fogo nos olhos da preguiça-real. $\mathrm{O}$ quatipuru tentou, mas voltou no meio do caminho. Finalmente aquele quatipuruzinho bem pequeno, taine, conseguiu subir. Jogou as formigas e a preguiça soltou o céu. A árvore caiu e a luz apareceu. Taine casou-se com Aicüna. (GRUBER, 2000, pg. 14)

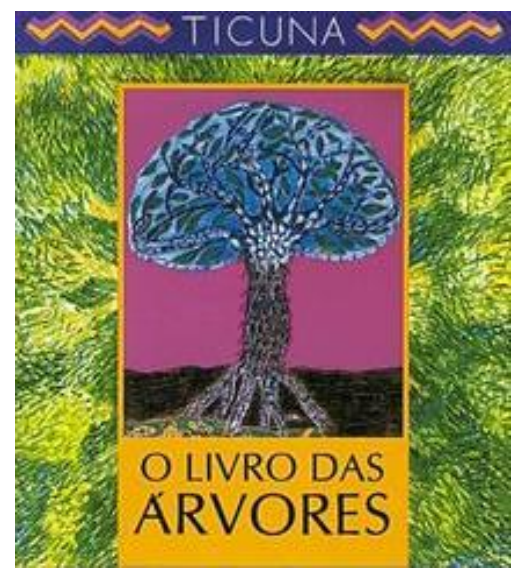

Figura 1 - Capa do livro O Livro das Árvores. Fonte: Acervo pessoal. 
Como no mito da samaumeira, as relações intrínsecas dos povos indígenas com seu meio constituem as cosmologias (ou epistemologias) dos povos originários. Criando uma analogia com a ocupação feita pelas crianças no parque da escola, espaço cotidianamente ocupado por elas e, portanto, que constitui o que elas são e sabem, o espaço é sujeito e não objeto na troca de saberes com as crianças, por isso, foi escolhido como espaço de pesquisa.
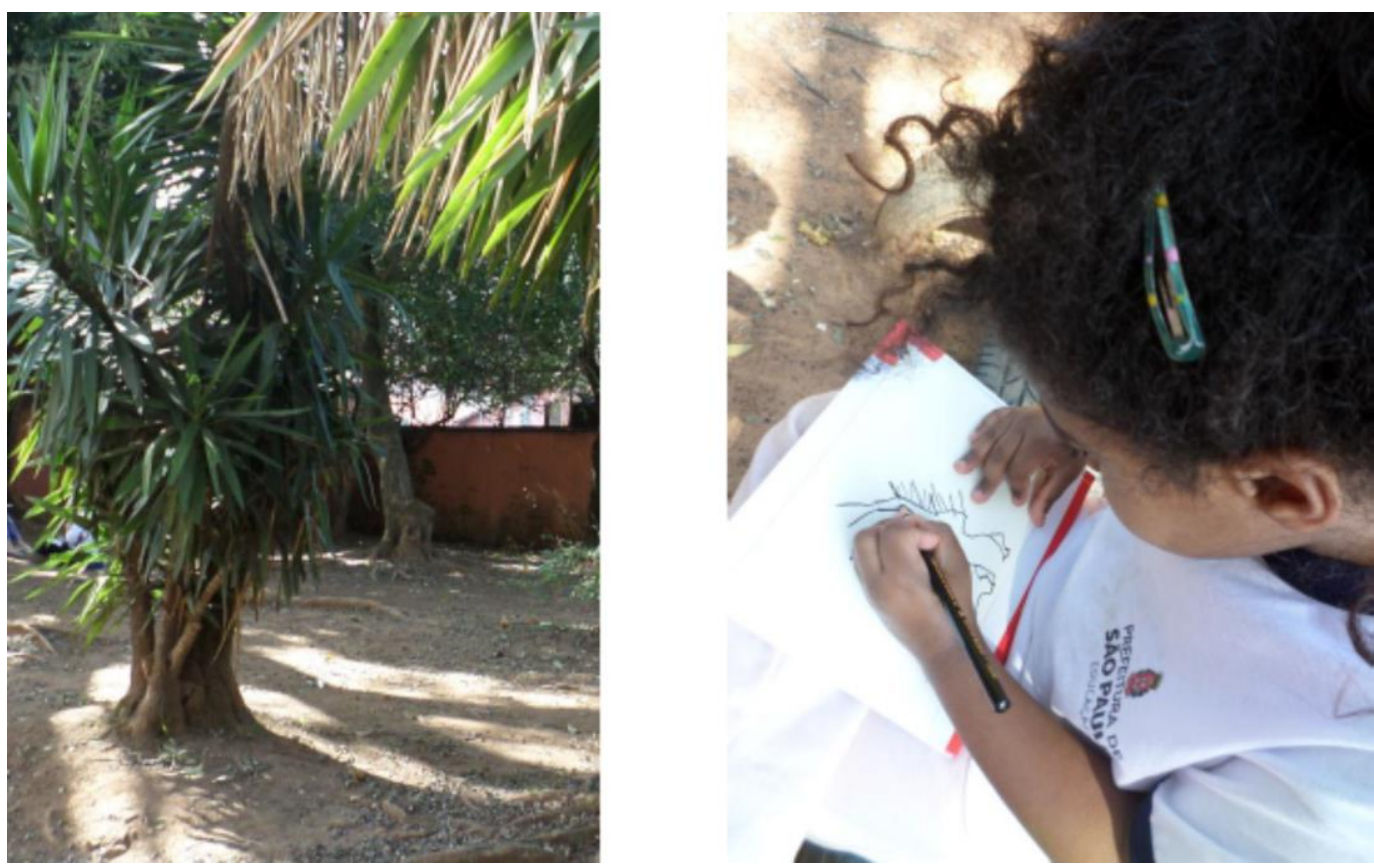

Figura 2 - Criança observando e desenhando no parque. Fonte: Acervo pessoal.

Da relação de sujeito-natureza e sujeito-pessoa, partiu-se do princípio de que a sabedoria de nossos povos originários está presente no conhecimento das características das árvores e o uso que se pode fazer de cada parte desses seres, como a utilização da madeira, das raízes, das folhas, sementes e frutos.

Inicialmente, foi proposto o registro das árvores através da fotografia, mas esse procedimento não cumpriu um dos objetivos principais da proposta, que era o de estimular um vínculo afetivo por meio da percepção visual-espacial. Por ser um processo rápido de registro, sem permitir uma relação mais longa com o objeto registrado, foi sugerido que escolhessem as árvores e fizessem desenhos de observação, atribuindo um nome de acordo com as características da planta: Amarelinha, Fofa, Peladinha, Super-árvore, Gege, Abacaxi, 
Pé de monstro, Tortinha, Tobogã. Os nomes dados pelas crianças revelaram características da árvore, da própria criança, das relações estabelecidas e de um universo fantástico.

Essa relação de ver além da materialidade do ser que enxergamos e sua energia vital, interfere no modo como nos vemos e vemos o outro, ou seja, suas características podem aparecer na organização e nomeação das brincadeiras como, por exemplo, os nomes das árvores.

Uma possibilidade de descentralização de uma realidade única, isto é, da razão europeia e capitalista, é pensar dialogicamente com as epistemes indígenas, que consideram o universo em sua totalidade e em uma rede complexa de relações que estruturam-se pela multiplicidade de nações onde coexistem maneiras distintas de pensar e de viver. Esta escolha não se trata de uma transposição ingênua de perspectivas de mundo, talvez esteja mais próximo do perspectivismo ameríndio de Viveiros de Castro (1996), que pensa "o modo como humanos, animais e espíritos vêem-se a si mesmos e aos outros seres do mundo", antes de mais nada, são efeitos das relações em que estão localizados e assim se definem, redefinem e se produzem na medida em que as relações que os constituem muda.

Os indígenas são nosso passado, presente e futuro. Não são somente passado, onde são tradicionalmente localizados nos registros escritos. Suas REexistências no mundo capitalista são a prova de que outra forma de viver é possível, pois revela possibilidades mais éticas de se relacionar com o meio e todos os seres. São uma alternativa para o capitalismo e o socialismo, e um exemplo de resistência à colonialidade que estrutura nossos imaginários e identidades. Uma civilização que depende da destruição do seu meio para a sua sobrevivência, tem muito o que aprender com as formas não-exploratórias e não-devastadoras dos povos indígenas de se relacionarem com o meio e todos os seres, encantados ou não.

Retomando a experiência do desenho de observação, em um processo dialógico, as educadoras anotavam as impressões das crianças, o que possibilitou, por meio da oralidade, registrar o conhecimento produzido por cada um. Abaixo, um exemplo de como essas informações foram sistematizadas: 

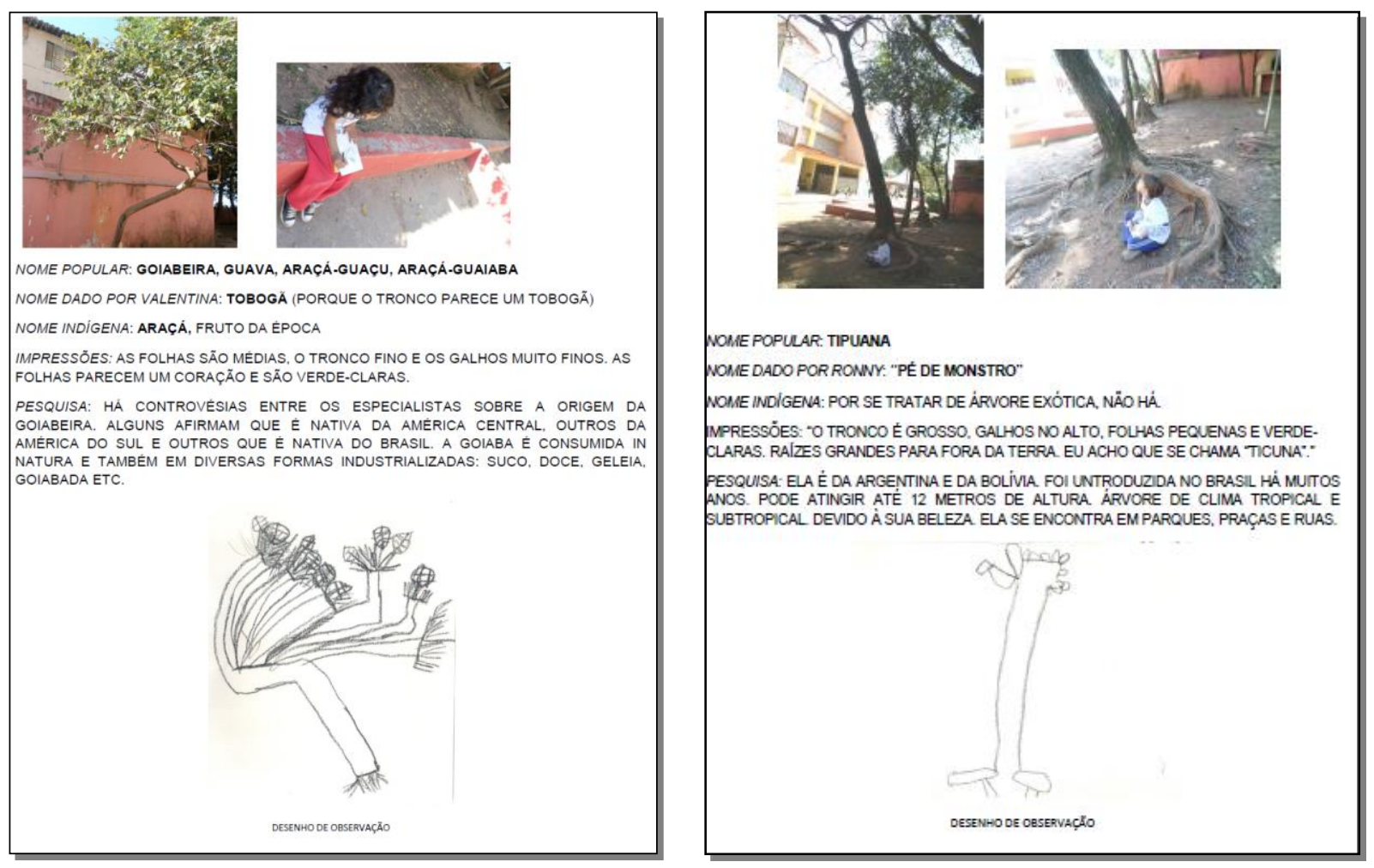

Figura 3 - Páginas do livro Árvores e Plantas do Amorim.

Fonte: Acervo pessoal.

No desenvolvimento do projeto, os nomes das árvores foram se revelando e as mudanças temporais que ocorriam nas folhas, flores e frutos foram acompanhadas. Nos horários de parque e recreio sempre havia um estudante compartilhando as transformações observadas.

Na perspectiva de enriquecer essa atividade de observação e descoberta, foi feita uma parceria com o etnobotânico e estudante de língua tupi Lucas Ciola, que organizou uma aula com as crianças, apresentando como se nomeiam as árvores em tupi. Em pequenos grupos, visitaram as árvores para observar suas características e aprender algumas "chaves" de como identificar as plantas na lógica indígena. 


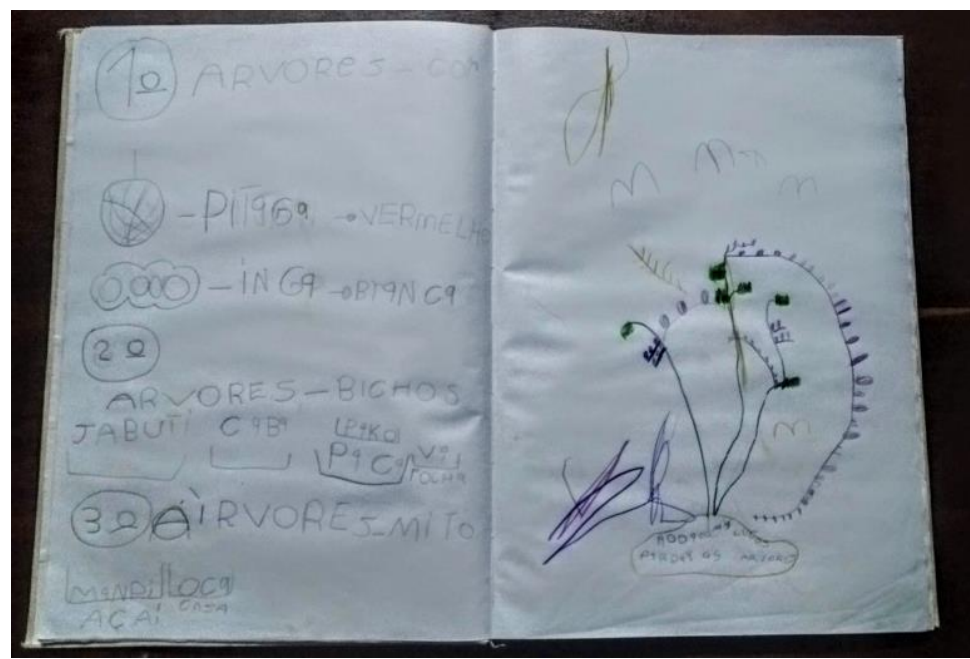

Figura 4 - Registro do livro Árvores e Plantas do Amorim, produzido a partir da aula de Lucas Ciola. Fonte: Acervo pessoal.

Os nomes das árvores viraram listas de palavras para as oficinas de leitura e escrita. Localizar cada árvore na planta da escola com o uso da ferramenta do Google Maps foi tarefa das oficinas de matemática. Cabe esclarecer que o projeto pedagógico da escola está organizado por oficinas e horários de pesquisa, ao invés de aulas expositivas ${ }^{6}$.

Para cada criança e sua árvore escolhida do parque, foi montada uma página com a foto da árvore e o desenho de observação realizado pela criança. As páginas tornaram-se cartazes que ficaram expostos nas árvores correspondentes no dia da Festa da Cultura, juntamente com um mapa mostrando a sua localização. Depois, foi montado artesanalmente o livro Árvores e Plantas do Amorim.

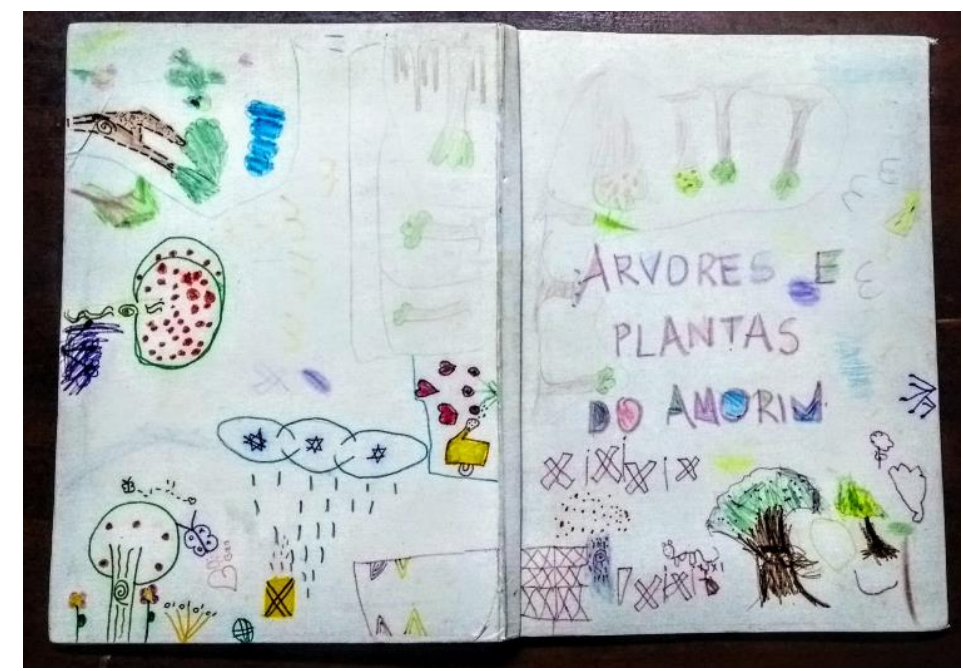

\footnotetext{
${ }^{6}$ Disponível em: 〈https://amorimlima.org.br/>. Acesso em: 16 mai. 2019.
} 
RELACult - Revista Latino-Americana de Estudos em Cultura e Sociedade

Revista Latinoamericana de Estudios en Cultura y Sociedad | Latin American Journal of Studies in Culture and Society V. 05, ed. especial, mai., 2019, artigo n ${ }^{\circ} 1556$ | claec.org/relacult | e-ISSN: 2525-7870

Figura 5 - Capa do livro artesanal Árvores e Plantas do Amorim, produzido pelas crianças e professoras Eliene O. Aleixo e Maria Lucia Padilha.

Fonte: Acervo pessoal.

Ainda no contexto desse projeto, foram realizados alguns estudos de meio, tais como: uma caminhada monitorada pela "Trilha do Jequitibá", no Parque da Previdência e uma visita à aldeia Guarani Krukutu, ambos na cidade de São Paulo.

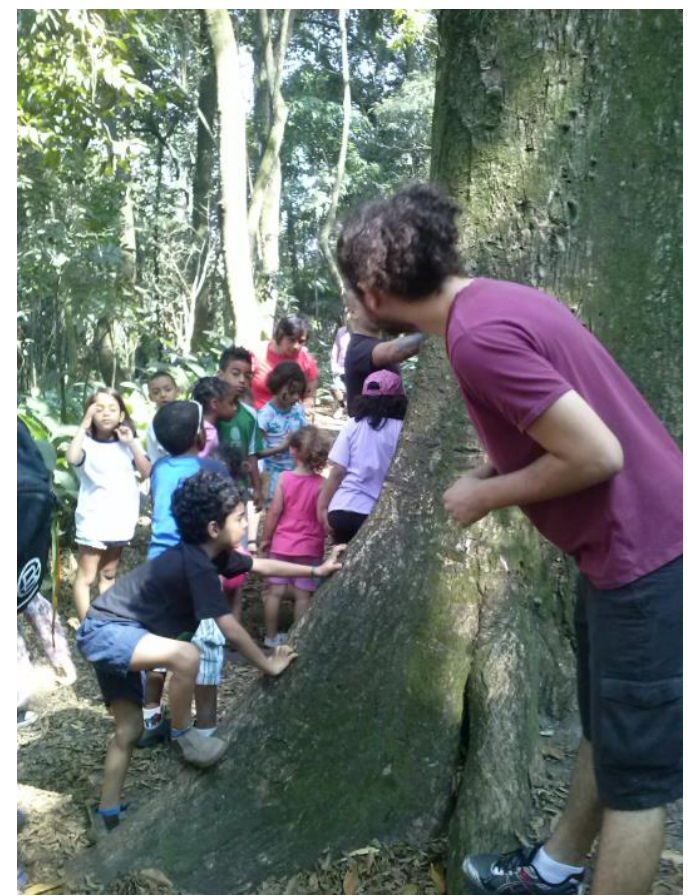

Figura 6 - Estudo de Campo: Visita ao Parque da Previdência, trilha do jequitibá. Fonte: Acervo pessoal.

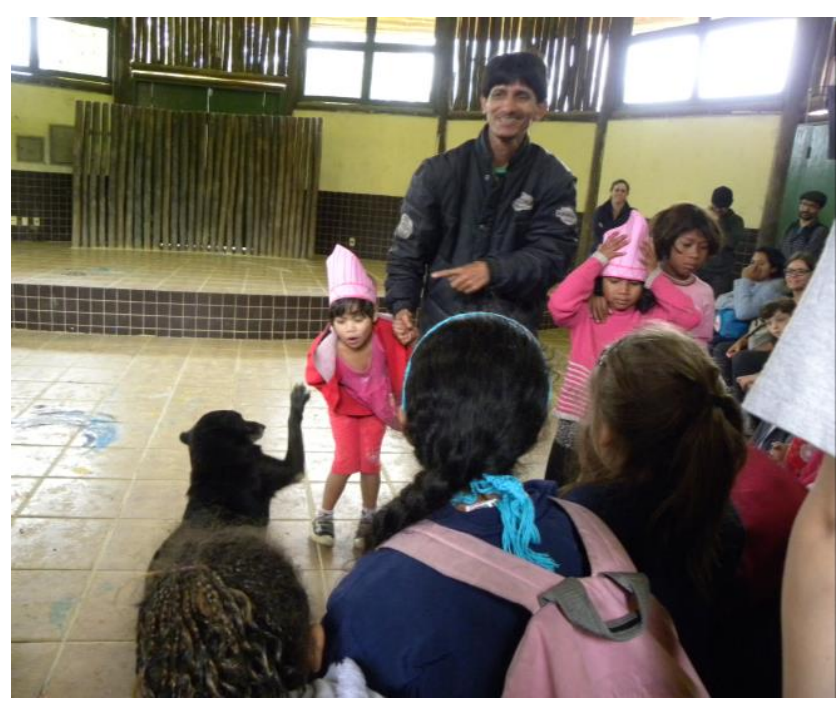

Figura 7 - Estudo de Campo: Visita à Aldeia Guarani Krukutu. Fonte: Acervo pessoal. 
Para concluir, consideramos que a experiência permitiu conhecer a complexidade da cosmologia Ticuna, desconstruindo a ideia do colonizador de que "índio é tudo igual" e valorizar o conhecimento que se estrutura a partir das vivências cotidianas das crianças. Em projeto de educação emancipadora, a valorização das sabedorias dos indivíduos em um contexto em que historicamente ratifica discursos dominantes, como é o espaço da escola, promove a insurgência dos grupos que são marginalizados na produção do conhecimento "fora da cartilha" como é o das crianças e dos povos originários.

No contexto escolar e na sociedade, em decorrência de uma lógica produtivista, as vozes das crianças muitas vezes são silenciadas, assim como as dos povos originários, o que não permite que esses sujeitos narrem as suas histórias e as façam fonte de conhecimento. Essa invisibilização compromete diretamente a formação das identidades e subjetividades, violentando e/ou anulando a existência social, histórica e cultural daqueles que não se enquadram nos padrões hegemonicamente aceitos socialmente, ou seja, de ser homem, branco, burguês e ocidental.

Nesse sentido, entendemos que esse projeto fez frente a esta condição subalternizada tanto de crianças e educadores quanto dos povos indígenas. Além disso, essa abordagem crítica contribuiu com a efetiva aplicação da Lei 11.645/08, que estabelece a obrigatoriedade do ensino da história e das culturas afro-brasileiras e indígenas na Educação Básica, uma política pública fundamental para uma educação plural que respeita a diversidade cultural dos povos do Brasil.

Para finalizar, defendemos que somente uma abordagem crítica das relações que permeiam a produção do conhecimento tanto de educadores como de educandos pode desestabilizar as relações de poder no espaço escolar, respeitando outras formas de ensinar e aprender além daquelas que hegemonicamente são reconhecidas. Aprender com os povos originários do Brasil, permite reestabelecer diálogos com os encantados, os seres fantásticos, a natureza e nós mesmos.

\section{Referências}

GRUBER, Jussara Gomes. O Livro das Árvores. 4. ed. Benjamin Constant, AM: Organização Geral dos Professores Ticuna Bilingues; São Paulo: Global, 2000. 
MALDONADO-TORRES, Nelson. Sobre la colonialidade del ser: contribuiciones al desarrollo de um concepto. In: CASTRO-GÓMEZ, Santiago e GROSFOGUEL, Ramón (Orgs). El giro decolonial. Reflexiones para uma diversidade epistémica más allá del capitalismo global. Bogotá: Universidade Javeriana-Instituo Pensar/Universidad CentralÍESCO/ Siglo del Hombre Editores, 2007.

OLIVEIRA, Luiz Fernandes. Educação e Militância Decolonial. Rio de Janeiro: Editora Selo Novo, 2018.

QUIJANO, Aníbal. Colonialidad del poder, eurocentrismo y América Latina. In: LANDER, E. (Org.). La colonialidad del saber: eurocentrismo y ciencias sociales. Perspectivas Latinoamericanas. Buenos Aires: Clacso, 2005, p. 227-277.

VIVEIROS DE CASTRO, Eduardo. Os pronomes cosmológicos e o perspectivismo ameríndio. In: Mana 2 (2), versão eletrônica, 1996. 